\title{
Self-consistent theory of finite Fermi systems vs Skyrme-Hartree-Fock method. Spherical nuclei.
}

\author{
E. E. Saperstein ${ }^{1}{ }^{2, a}$ and S. V. Tolokonnikov ${ }^{1},^{3}$ \\ ${ }^{1}$ National Research Centre "Kurchatov Institute", Moscow, 123182 Russia \\ ${ }^{2}$ National Research Nuclear University MEPhl, Moscow, 115409 Russia \\ ${ }^{3}$ Moscow Institute of Physics and Technology, Dolgoprudny, Moscow Region, 141700 Russia
}

\begin{abstract}
Recent results of the Fayans energy density functional (EDF) for spherical nuclei are reviewed. A comparison is made with predictions of several Skyrme EDFs. The charge radii and characteristics of the first $2^{+}$excitations in semi-magic nuclei are briefly discussed. The single-particle spectra of doubly magic nuclei are considered in more detail. The phonon-particle coupling effects are analyzed including the tadpole term.
\end{abstract}

\section{Introduction}

In this century, the Hartree-Fock (HF) method with Skyrme forces [1] dominates in the theoretical description of ground states of heavy nuclei. This method is often considered as a version of the Energy Density Functional (EDF) method by Kohn and Sham [2], which is based on the Hohenberg-Kohn theorem [3], stating that the ground state energy $E_{0}$ of a Fermi system is a functional of its density $\rho$. The Skyrme HF ansatz leads to a rather simple form of the nuclear EDF. The same is true for the HF method with Gogny force [4] which becomes again popular last decade. For completeness, we mention also the relativistic mean field (RMF) model, see recent Ref. [5] and links there, and a newly developed self-consistent approach, known as a method BCPM (Barcelona - Catania Paris - Madrid) [6, 7].

We use an alternative EDF method developed by Fayans with coauthors [8-11] with more sophisticated density dependence. The main, in-volume term of the Fayans EDF can be symbolically written as

$$
\mathcal{E}(\rho)=\frac{a \rho^{2}}{2} \frac{1+\alpha \rho^{\sigma}}{1+\gamma \rho^{\sigma}},
$$

where $\rho(\mathbf{r})$ is the total nuclear density, $a, \alpha, \sigma, \gamma$ being parameters. The corresponding term of the Skyrme EDF corresponds to $\gamma=0$ in this relation. The use of the bare mass $m^{*}=m$ is another peculiarity of the Fayans EDF. Both the features of the Fayans method are closely related to the self-consistent Theory of Finite Fermi Systems (TFFS) and represent, in a hidden form, energy dependence effects inherent to this approach. The latter is based on the general principles of the TFFS [12] with inclusion of the TFFS self-consistency relations [13]. The final version of this approach $[14,15]$ was formulated in terms of the

\footnotetext{
a e-mail: saper43〈protect $\_7 @$ mail.ru
}

quasiparticle Lagrangian $\mathcal{L}_{q}$, which is constructed to produce the quasiparticle mass operator $\Sigma_{q}\left(\mathbf{r}, k^{2} ; \varepsilon\right)$. By definition, the latter coincides with the exact mass operator $\Sigma\left(\mathbf{r}, k^{2} ; \varepsilon\right)$ at the Fermi surface. In the mixed coordinatemomentum representation it depends linearly on the momentum squared $k^{2}$ and the energy $\varepsilon$ as well [12].

In the TFFS, the effective mass is a product $m^{*}=m_{k}^{*} \cdot m_{E}^{*}$ of the "k-mass" and the "E-mass". The two effects compensate each other almost exactly [15] resulting in $m^{*} \simeq 1$ which justifies the Fayans choice of the bare mass instead of the effective one. The EDF of the self-consistent TFFS is found from the Lagrangian $\mathcal{L}_{q}$ according the canonical rules. It includes implicitly the $Z$-factor

$$
Z(\mathbf{r})=\left(1-\left(\frac{\partial \Sigma}{\partial \varepsilon}\right)\right)_{0}^{-1},
$$

where the index 0 means that the energy and momentum variables are taken at the Fermi surface. Its density dependence can be found explicitly [15]

$$
Z(\mathbf{r})=2\left(1+\sqrt{1-4 C_{0} \lambda_{02} \rho(\mathbf{r}) / \varepsilon_{\mathrm{F}}^{0}}\right)^{-1},
$$

where $C_{0}=\left(d n / d \varepsilon_{\mathrm{F}}^{0}\right)^{-1}=\pi^{2} / m p_{\mathrm{F}}^{0}$ is the usual TFFS normalization factor, inverse density of states at the Fermi surface, and the dimensionless parameter $\lambda_{02}$ determines the $Z$-factor of nuclear matter $Z_{0}$. In the result, the density dependence of the EDF becomes rather complicated [15]. Fayans with coauthors found [8] that, in a wide density region, it can be approximated with high accuracy by a more simple expression (1). Thus, the Fayans EDF method can be interpreted as a version of the self-consistent TFFS.

Till this year, Ref. [16], all self-consistent calculations with Fayans functionals were carried out for spherical nuclei only. It proved out successful in systematic description of nuclear magnetic [17, 18] and quadrupole [19-21] moments and nuclear radii [22] as well. In the latter case, agreement with the data is better than that in all SHF calculations we know. There are two examples more where 
we may compare the two approaches directly. The energies and $B(E 2)$ values for the first $2^{+}$excitations in semimagic nuclei $[19,23]$ is one of them. They are described with the Fayans EDF much better than in the known SHF calculations with SkM* and SLy4 EDFs [24]. The singleparticle energies (SPEs) $\varepsilon_{\lambda}$ in seven doubly magic nuclei, for which the experimental spectra are known [25], is another example [26]. The PC corrections to $\varepsilon_{\lambda}$ were found self-consistently with account for the tadpole diagram. A systematic comparison to the SHF predictions with a popular HFB-17 EDF [27] was carried out. Even at the level of the mean field theory, the Fayans EDF results are significantly better. Inclusion of the PC corrections makes the agreement better yet.

The article is organized as follows. Sec. 2 contains a brief comparison with several Skyrme EDFs of predictions for the charge radii and characteristics of the first $2^{+}$excitations in semi-magic nuclei. Sec. 3 is devoted to the description of SPEs in doubly magic nuclei. Sec. 4 contains conclusions.

\section{Charge radii and characteristics of $2_{1}^{+}$ levels in semi-magic nuclei}

In Ref. [22], systematic self-consistent calculations of charge radii $R_{\mathrm{ch}}$ were made on the base of the DF3-a EDF. Deformed nuclei were also included into analysis, with an approximate taking into account the deformation effect. Agreement with the data on the level of $0.01 \mathrm{fm}$ was achieved, noticeably better than for SLy4 and HFB17 Skyrme EDFs taken for comparison. The HFB-17 predictions were taken from [28], whereas for the SLy4 EDF calculations were made in [22]. As an example, the lead charge radii are displayed in Fig. 1. We see that the Fayans EDF, indeed, describes the radii perfectly well. The HFB17 one reproduces the data reasonably for heavy $\mathrm{Pb}$ isotopes but fails systematically for $A<190$ where the disagreement reaches $0.1 \mathrm{fm}$. The reason for this is that this EDF erroneously predicts rather strong stable deformation for the light $\mathrm{Pb}$ isotopes which leads to a significant increase of $R_{\mathrm{ch}}$ values. This problem is discussed in detail in [16]. The $R_{\mathrm{ch}}$ values predicted with SLy4 are systematically higher than the experimental ones at approximately $0.03 \mathrm{fm}$. This is a typical scale of accuracy in describing nuclear radii for different Skyrme EDFs.

A high accuracy of reproducing the charge radii may be linked to more adequate density dependence of the Fayans EDF compared to the Skyrme one. Indeed, if we denote the average error in describing the binding energies as $\overline{\delta E}$ and that for the charge radii as $\overline{\delta R_{\mathrm{ch}}}$, these quantities should be, due to the Hohenberg-Kohn theorem [3], proportional to each other,

$$
\overline{\delta R_{\mathrm{ch}}}=\alpha \overline{\delta E},
$$

where the coefficient $\alpha$ depends on the functional we use. This almost obvious relation can be proved with such simple consideration. Let we have an exact $\operatorname{EDF} \mathcal{E}_{0}(\rho)$ and add to it a small addendum $\delta \mathcal{E}_{0}=\lambda f(\rho)$, where the coefficient is small, $\lambda \ll 1$. Obviously, we get $\overline{\delta E}=$ $\lambda \int d \mathbf{r} f(\rho(\mathbf{r}))=\lambda a$; then the change of the mean field

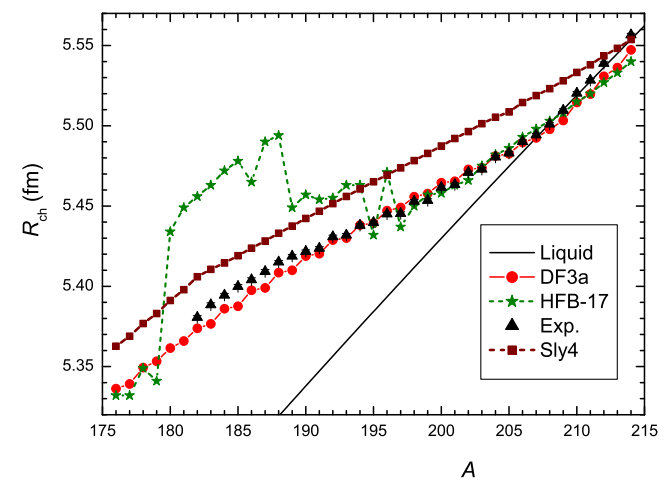

Figure 1. (Color online) Charge radii in lead isotopes. Solid line shows the Liquid Drop model predictions.

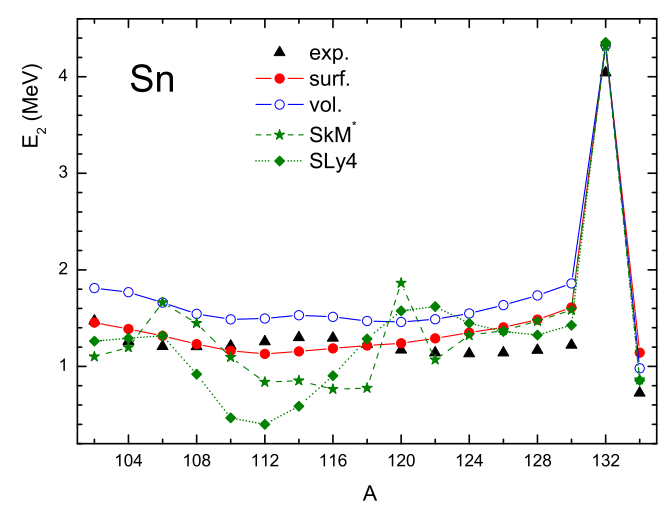

Figure 2. (Color online) Excitation energies $\omega\left(2_{1}^{+}\right)$for tin isotopes. Predictions for the SkM* and SLy4 force are taken from [24].

$\delta U=\delta f / \delta \rho$ is proportional to $\lambda$. Let us find the change of the density $\rho$ in the first order of the perturbation theory $\delta U$. It can be easily seen that $\delta \rho$ and the corresponding change of the radius $\overline{\delta R_{\mathrm{ch}}}$ are also proportional to $\lambda$, $\overline{\delta R_{\mathrm{ch}}}=\lambda b$. In the result, we obtain Eq. (4) with $\alpha=b / a$. As a rule, a fine tuning of the EDF parameters is performing by focusing mainly on reproduction of the nuclear masses within a minimal value of $\overline{\delta E}$. In this case, the accuracy of reproducing the charge radii is proportional to the coefficient $\alpha$. As the analysis of [22] showed, for the Fayans EDF this coefficient is less than those of the HFB17 and SLy4 functionals. This observation may be linked to more sophisticated density dependence of Fayans functional, which allows to incorporate implicitly the energy dependence effects.

In Ref. [19], excitation energies $\omega_{2}$ and transition probabilities $B(E 2)$ of the first $2^{+}$excitations in even tin and lead isotopes as well as the quadrupole moments of odd neighbors of these isotopes were calculated within the self-consistent TFFS with the use of the DF3-a EDF. The effect of the density dependence of the effective pairing interaction was analyzed by comparing results obtained by solving the QRPA equations with volume and surface pairing. The effect was found to be noticeable giving evidences in favor of the surface pairing. For example, the $2^{+}$-energies are systematically higher at $200-400 \mathrm{keV}$ for 


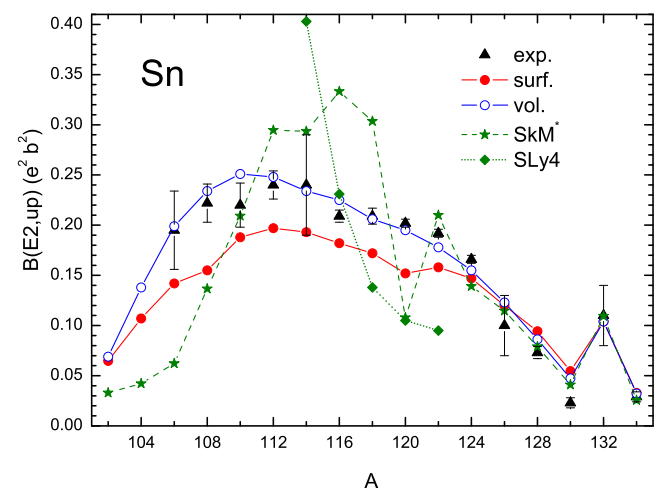

Figure 3. (Color online) $B(E 2$, up) values for tin isotopes. Predictions for the SkM* and SLy4 force are taken from [24].

the volume paring as compared with the surface pairing case, the latter being on average better. Obtained results were compared with predictions of [24] for the Skyrme EDFs SkM* and SLy4. For tin isotopes, the comparison of $\omega_{2}$ and $B(E 2)$ values is given in Figs. 2 and 3, respectively. On the average, predictions of the Fayans EDF with both models for pairing reasonably agrees with the data, significantly better than those for both the Skyrme EDFs. Among the latter, the SkM* EDF turns out to be more successful. For the SLy4 EDF, the ${ }^{112} \mathrm{Sn}$ nucleus and its neighbors are very close to the point of the quadrupole instability, in contradiction with the data.

\section{Single-particle spectra of magic nuclei}

The major part of modern calculations with the Fayans functional in spherical nuclei are made with the EDF DF3a. This is a version [29] of the EDF DF3 [9, 11] with modified spin-dependent parameters, the spin-orbit $\kappa, \kappa^{\prime}$ and the first spin harmonics $g_{1}, g_{1}^{\prime}$ which play the role of the effective tensor forces. The DF3 and DF3-a EDFs both contain 3 non-zero spin-dependent parameters, specifically, the value of $g_{1}=0$ is putted. The bulk of the data [25] for SPEs contains 65 spin-orbit differences which permits to try to find an optimal set of the spin-dependent parameters of the EDF. Such a set DF3-b, with $g_{1} \neq 0$, was found in [26], but the overall description of these differences turned out only a bit better than for DF3 or DF3-a sets. Moreover, the average accuracy in reproducing SPEs $\varepsilon_{\lambda}$, see Table 1, for DF3-b EDF is the same as for DF3 and only a little better than for the DF3-a EDF. The latter was chosen for systematic calculations as far as it previously proved to be successful in description of different nuclear phenomena $[19,20,29]$. For comparison, we found the SPEs of all nuclei under consideration for the Skyrme EDF HFB-17 [27] which is a record-holder in reproducing nuclear masses.

Figures 4 and 5 show the comparison to the data [25] of predictions of all the EDFs under discussion for the SPEs in ${ }^{208} \mathrm{~Pb}$ for neutrons and protons, respectively. We see that both HFB-17 spectra, especially the neutron one, are too expanded. This is the result of influence of the effective mass $m^{*}<m$. The analysis in Ref. [30] confirmed a pref-
Table 1. Average deviations $\left\langle\delta \varepsilon_{\lambda}\right\rangle_{\mathrm{rms}}(\mathrm{MeV})$ of the theory predictions for the single-particle energies from the experimental values for magic nuclei.

\begin{tabular}{cccccc}
\hline \hline Nucleus & $N$ & DF3-b & DF3-a & DF3 & HFB17 \\
\hline${ }^{40} \mathrm{Ca}$ & 14 & 1.08 & 1.25 & 1.35 & 1.64 \\
${ }^{48} \mathrm{Ca}$ & 12 & 0.89 & 1.00 & 1.01 & 1.70 \\
${ }^{56} \mathrm{Ni}$ & 14 & 1.00 & 0.97 & 0.85 & 1.40 \\
${ }^{78} \mathrm{Ni}$ & 11 & 1.24 & 1.41 & 1.09 & 1.32 \\
${ }^{100} \mathrm{Sn}$ & 13 & 1.09 & 1.17 & 1.01 & 1.56 \\
${ }^{132} \mathrm{Sn}$ & 17 & 0.58 & 0.66 & 0.55 & 1.15 \\
${ }^{208} \mathrm{~Pb}$ & 24 & 0.44 & 0.51 & 0.43 & 1.15 \\
\hline Total & 105 & 0.89 & 0.98 & 0.89 & 1.40 \\
\hline \hline
\end{tabular}

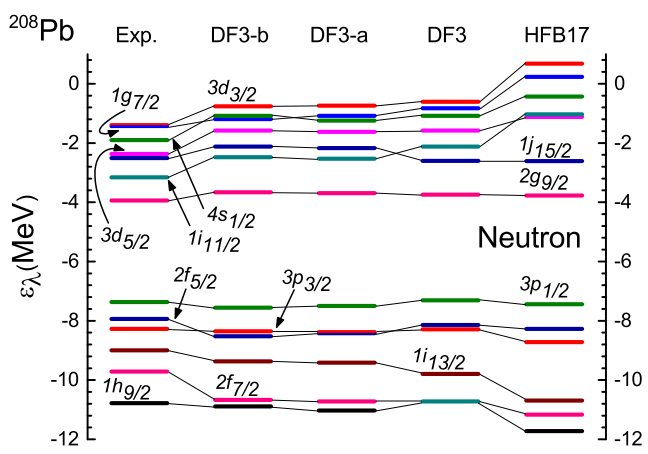

Figure 4. (Color online) Neutron single-particle levels in ${ }^{208} \mathrm{~Pb}$. Experimental data from [25].

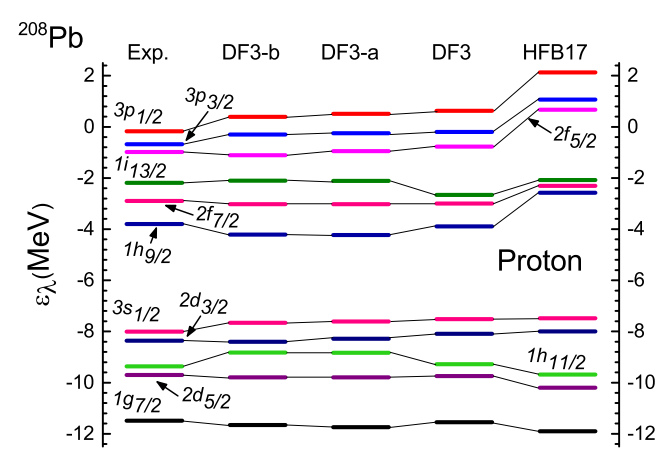

Figure 5. (Color online) Proton single-particle levels in ${ }^{208} \mathrm{~Pb}$. Experimental data from [25].

erence of the choice of the bare mass for describing SPEs with Skyrme EDFs without PC corrections.

Let us go to the PC contributions to SPEs. Accounting for PC effects, the equation for SPEs and corresponding wave functions can be written as

$$
\left(\varepsilon-H_{0}-\delta \Sigma^{\mathrm{PC}}(\varepsilon)\right) \phi=0,
$$

where $H_{0}$ is the quasiparticle Hamiltonian with the spectrum $\varepsilon_{\lambda}^{(0)}$ and $\delta \Sigma^{\mathrm{PC}}$ is the $\mathrm{PC}$ correction to the quasiparticle mass operator. After expanding this term in the vicinity of $\varepsilon=\varepsilon_{\lambda}^{(0)}$ one finds

$$
\varepsilon_{\lambda}=\varepsilon_{\lambda}^{(0)}+Z_{\lambda}^{\mathrm{PC}} \delta \Sigma_{\lambda \lambda}^{\mathrm{PC}}\left(\varepsilon_{\lambda}^{(0)}\right),
$$




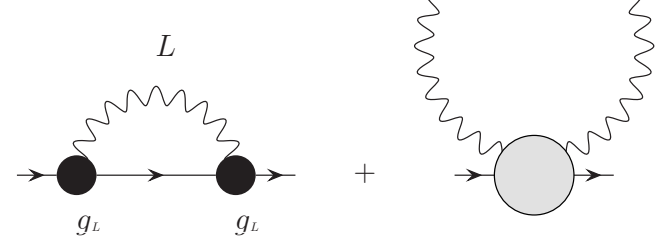

Figure 6. PC corrections to the mass operator. The gray circle denotes the "tadpole" term.

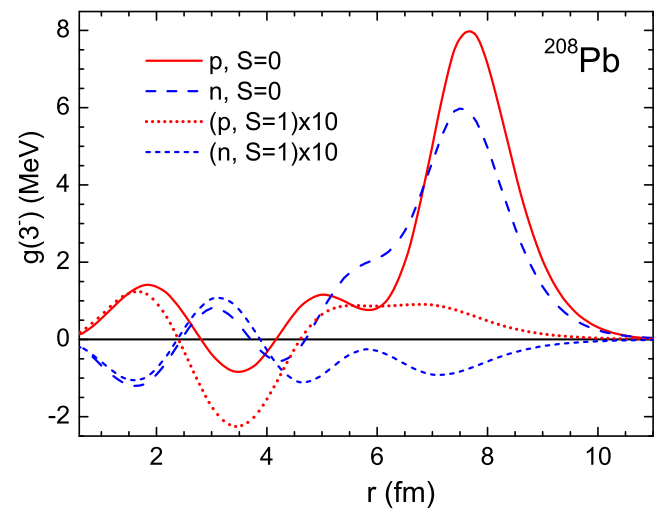

Figure 7. (Color online) The vertex $g_{L}$ for the $3_{1}^{-}$state in ${ }^{208} \mathrm{~Pb}$.

with obvious notation. Here $Z^{\mathrm{PC}}$ denotes the $Z$-factor due to the PC effects, i.e. that found from Eq. (2) with substitution of $\delta \Sigma^{\mathrm{PC}}(\varepsilon)$ instead of the main mass operator $\Sigma(\varepsilon)$. Remember that in the TFFS the corresponding $Z$-factor is included in the quasiparticle Hamiltonian $H_{0}$.

The PC correction to the mass operator is displayed in Fig. 6, where $g_{L}$ is the vertex for creating the $L$-phonon. In magic nuclei, it obeys the equation [12]

$$
g_{L}(\omega)=\mathcal{F} A(\omega) g_{L}(\omega),
$$

where $A(\omega)=\int G(\varepsilon+\omega / 2) G(\varepsilon-\omega / 2) d \varepsilon /(2 \pi i)$ is the particle-hole propagator, $G(\varepsilon)$ being the one-particle Green function. In obvious symbolic notation, the pole diagram corresponds to $\delta \Sigma^{\text {pole }}=\left(g_{L}, D_{L} G g_{L}\right)$, where $D_{L}(\omega)$ is the phonon $D$-function.

All the low-lying phonons we consider have natural parity. In this case, the vertex $g_{L}$ possesses even $T$-parity. It is a sum of two components with spins $S=0$ and $S=1$, respectively,

$$
g_{L}=g_{L 0}(r) T_{L L 0}(\mathbf{n}, \alpha)+g_{L 1}(r) T_{L L 1}(\mathbf{n}, \alpha)
$$

where $T_{J L S}$ stand for the usual spin-angular tensor operators. The operators $T_{L L 0}$ and $T_{L L 1}$ have opposite $T$-parities, hence the spin component should be the odd function of the excitation energy, $g_{L 1} \propto \omega_{L}$. For the ghost dipole, $L=1$ and $\omega_{1}=0$, Eq. (7), due to the TFFS self-consistency relation [13], has the exact solution

$$
g_{1}(\mathbf{r})=\alpha_{1}(d U(r) / d r) Y_{1 M}(\mathbf{n}),
$$

where $\alpha_{1}=1 / \sqrt{2 \omega B_{1}}, B_{1}=3 m A / 4 \pi$ is the Bohr-Mottelson (BM) mass coefficient [31] and $U(r)$ is the central part of the mean-field potential generated by the energy functional.

The second, tadpole, term in Fig. 6 is

$$
\delta \Sigma^{\mathrm{tad}}=\int \frac{d \omega}{2 \pi i} \delta_{L} g_{L} D_{L}(\omega),
$$

where $\delta_{L} g_{L}$ can be found [15] by variation of Eq. (7) in the field of the $L$-phonon

$$
\begin{aligned}
\delta_{L} g_{L} & =\delta_{L} \mathcal{F} A\left(\omega_{L}\right) g_{L}+\mathcal{F} \delta_{L} A\left(\omega_{L}\right) g_{L} \\
& +\mathcal{F} A\left(\omega_{L}\right) \delta_{L} g_{L} .
\end{aligned}
$$

The quantity $\delta_{L} A$ can be readily obtained by variation of each Green function in the particle-hole propagator $A$ in field $g_{L}$ induced by the $L$-phonon. The explicit expression for the variation $\delta_{L} \mathcal{F}$ can not be found within the TFFS as in this approach the Landau-Migdal amplitude $\mathcal{F}$ is introduced as a phenomenological quantity. In [15], the ansatz was proposed,

$$
\delta_{L} \mathcal{F}=\frac{\delta \mathcal{F}(\rho)}{\delta \rho} \delta \rho_{L},
$$

where

$$
\delta \rho_{L}=A_{L} g_{L}
$$

is the transition density for excitation of the $L$-phonon. The phonon $D$-function appears in Eq. (10) after connecting two wavy phonon ends in Eq. (11). This corresponds to averaging of the product of two boson (phonon) operators $B_{L}^{+} B_{L}$ over the ground state of the nucleus with no phonons.

The $L$-phonon excitation energies $\omega_{L}$ and creation amplitudes $g_{L}(\mathbf{r})$ were found by solving Eq. (7) in a selfconsistent way with the DF3-a functional. In more detail, the procedure is described in [19]. All the $L$-phonons we consider are the surface vibrations which belong to the Goldstone mode corresponding to the spontaneous breaking of the translation symmetry in nuclei [15]. The coordinate form of their creation amplitudes $g_{L}(\mathbf{r})$ is very close to that, Eq. (9), for the ghost $1^{-}$phonon, which is the lowest energy member of this mode:

$$
g_{L}(r)=\alpha_{L}(d U / d r)+\chi_{L}(r)
$$

where the in-volume correction $\chi_{L}(r)$ is rather small.

The smallness of the in-volume component $\chi_{L}$ is demonstrated in Fig. 7 for the $3_{1}^{-}$state in ${ }^{208} \mathrm{~Pb}$ which is the most collective one among the surface vibrations and plays the main role in PC corrections for this nucleus. The small spin components $S=1$ are also displayed. To make them distinguishable, they are multiplied by the factor of 10. The smallness of the spin components is typical for $L$-phonons with a high collectivity. The first, surface term on the right-hand side of Eq. (14) corresponds to the BM model for the surface vibrations [31], the amplitude $\alpha_{L}$ being related to the dimensionless BM amplitude $\beta_{L}$ as follows: $\alpha_{L}=R \beta_{L}$, where $R=r_{0} A^{1 / 3}$ is the nucleus radius, and $r_{0}=1.2 \mathrm{fm}$.

If one neglects in-volume contributions, the tadpole PC term (10) can be reduced to a very simple form 
Table 2. Pole and tadpole contributions (MeV) to PC corrections from $33_{1}^{-}$-state to neutron SPEs in ${ }^{208} \mathrm{~Pb}$.

\begin{tabular}{cccc}
\hline \hline$\lambda$ & $\delta \varepsilon_{\lambda}^{\text {pole }}$ & $\delta \varepsilon_{\lambda}^{\text {tad }}$ & $\delta \varepsilon_{\lambda}$ \\
\hline $3 d_{3 / 2}$ & -0.150 & 0.012 & -0.137 \\
$2 g_{7 / 2}$ & -0.142 & 0.061 & -0.081 \\
$4 s_{1 / 2}$ & -0.134 & 0.016 & -0.118 \\
$3 d_{5 / 2}$ & -0.147 & 0.023 & -0.124 \\
$1 j_{15 / 2}$ & -0.708 & 0.204 & -0.504 \\
$1 i_{11 / 2}$ & -0.058 & 0.198 & 0.140 \\
$2 g_{9 / 2}$ & -0.244 & 0.076 & -0.167 \\
$3 p_{1 / 2}$ & -0.220 & 0.053 & -0.167 \\
$2 f_{5 / 2}$ & -0.186 & 0.094 & -0.092 \\
$3 p_{3 / 2}$ & -0.205 & 0.056 & -0.149 \\
$1 i_{13 / 2}$ & 0.057 & 0.211 & 0.269 \\
$2 f_{7 / 2}$ & 0.724 & 0.091 & 0.815 \\
$1 h_{9 / 2}$ & -0.014 & 0.197 & 0.184 \\
\hline \hline
\end{tabular}

Table 3. Pole and tadpole contributions (MeV) to PC corrections from $3_{1}^{-}$-state to proton SPEs in ${ }^{208} \mathrm{~Pb}$.

\begin{tabular}{cccc}
\hline \hline$\lambda$ & $\delta \varepsilon_{\lambda}^{\text {pole }}$ & $\delta \varepsilon_{\lambda}^{\text {tad }}$ & $\delta \varepsilon_{\lambda}$ \\
\hline $3 p_{1 / 2}$ & -0.375 & 0.153 & -0.222 \\
$3 p_{3 / 2}$ & -0.371 & 0.152 & -0.219 \\
$2 f_{5 / 2}$ & -0.278 & 0.168 & -0.110 \\
$1 i_{13 / 2}$ & -0.534 & 0.266 & -0.268 \\
$2 f_{7 / 2}$ & -0.409 & 0.168 & -0.240 \\
$1 h_{9 / 2}$ & -0.054 & 0.222 & 0.168 \\
$3 s_{1 / 2}$ & -0.310 & 0.143 & -0.167 \\
$2 d_{3 / 2}$ & -0.241 & 0.146 & -0.095 \\
$1 h_{11 / 2}$ & -0.017 & 0.246 & 0.229 \\
$2 d_{5 / 2}$ & 0.435 & 0.147 & 0.582 \\
$1 g_{7 / 2}$ & -0.271 & 0.197 & -0.074 \\
\hline \hline
\end{tabular}

$$
\delta \Sigma_{L}^{\mathrm{tad}}=\frac{\alpha_{L}^{2}}{2} \frac{2 L+1}{3} \Delta U(r) .
$$

The general consideration of the tadpole term within the not-self-consistent TFFS, with solving Eq. (11), was carried out in [32]. It was found that the in-volume corrections to Eq. (15) are, indeed, small for heavy nuclei, e.g., for ${ }^{208} \mathrm{~Pb}$. At the same time, for light nuclei, e.g., ${ }^{40,48} \mathrm{Ca}$, the accurate solution of Eq. (11) diminishes the approximate value (15) for the tadpole term by $\simeq 30 \%$.

Following to [26], we neglect the in-volume corrections for all nuclei considered. To find the phonon amplitudes $\alpha_{L}$, we use the definition $\alpha_{L}^{\tau}=g_{L}^{\tau \text {,max }} /(d U / d r)^{\tau \text {,max }}$, with obvious notation. It should be noted that the values of $\alpha_{L}^{n}$ and $\alpha_{L}^{p}$ are always very close to each other and to that which follows from the BM model formula for $B(E L)$ : $B(E L)_{\mathrm{BM}}=(3 Z / 4 \pi)^{2} \beta_{L}^{2} R^{2 L}[31]$, where the dimensionless $\mathrm{BM}$ phonon creation amplitude $\beta_{L}$ is related to that used by us as $\alpha_{L}=\beta_{L} R / \sqrt{2 L+1}, R=1.2 A^{1 / 3}$. For example, for the $3_{1}^{-}$state in ${ }^{208} \mathrm{~Pb}$ we have: $\alpha_{L}^{n}=0.32 \mathrm{fm}, \alpha_{L}^{p}=0.33 \mathrm{fm}$, and $\alpha_{L}^{\mathrm{BM}}=0.30 \mathrm{fm}$.

A comparison of the pole and tadpole PC corrections to neutron and proton SPEs, induced by the $3_{1}^{-}$state in
Table 4. PC effect on average deviations $\left\langle\delta \varepsilon_{\lambda}\right\rangle_{\mathrm{rms}}(\mathrm{MeV})$ of the theory predictions for SPEs from the experimental values for the DF3-a functional.

\begin{tabular}{cccc}
\hline Nucleus & $N$ & DF3-a+PC & DF3-a \\
\hline${ }^{40} \mathrm{Ca}$ & 14 & 1.30 & 1.25 \\
${ }^{48} \mathrm{Ca}$ & 12 & 1.05 & 1.00 \\
${ }^{56} \mathrm{Ni}$ & 14 & 0.98 & 0.97 \\
${ }^{78} \mathrm{Ni}$ & 11 & 1.34 & 1.41 \\
${ }^{100} \mathrm{Sn}$ & 13 & 1.21 & 1.17 \\
${ }^{132} \mathrm{Sn}$ & 17 & 0.63 & 0.66 \\
${ }^{208} \mathrm{~Pb}$ & 24 & 0.38 & 0.51 \\
\hline total & 105 & 0.97 & 0.98 \\
\hline
\end{tabular}

${ }^{208} \mathrm{~Pb}$, are given in Tables 2 and 3, respectively. The tadpole term is always positive, whereas the pole one is, as a rule, negative, and the two contributions are of the opposite sign. The magnitude of the tadpole term is, as a rule, less than the pole one, but comparable with the latter. Especially, this is true for protons. Therefore the sum is often essentially less than the pole term alone. A typical suppression of the pole contribution is of $30-50 \%$, but there are cases of a stronger suppression, e.g. $2 d_{3 / 2}$ and $1 g 7 / 2$ proton states. Moreover, there are several cases when the tadpole term dominates: the neutron $1 i_{11 / 2}$ and $1 h_{9 / 2}$ states and the proton $1 h_{9 / 2}$ and $1 h_{11 / 2}$ ones. In these cases, the total correction is of the opposite sign as compared with the pole term. The general conclusion is that one overestimates the PC correction to SPEs neglecting the tadpole term, and often it is better to omit it completely than consider the pole term alone.

The total PC effect to SPEs of all doubly magic nuclei under consideration is shown in Table 4. We see that it makes the agreement essentially better for ${ }^{208} \mathrm{~Pb}$ but a bit less good in the light nuclei. The latter is explained mainly with the use the approximation (15) for the tadpole term. According to [32], the exact consideration for ${ }^{40,48} \mathrm{Ca}$ diminishes the tadpole term by approximately $30 \%$. Such a correction should lead to a better agreement with the experimental SPEs. Let us stress once more a high accuracy of reproducing the experimental SPEs in ${ }^{208} \mathrm{~Pb}:\left\langle\delta \varepsilon_{\lambda}\right\rangle_{\mathrm{rms}}=0.38(\mathrm{MeV})$. Indeed, the corresponding result [33], obtained within the RMF approach, is $\left\langle\delta \varepsilon_{\lambda}\right\rangle_{\mathrm{rms}}=0.85 \mathrm{MeV}$.

\section{Conclusions}

Recent studies with the Fayans EDF for spherical nuclei are reviewed and compared to predictions of several Skyrme EDFs. The charge radii and the characteristics of the first $2^{+}$excitations in semi-magic nuclei are briefly discussed. The accuracy of reproducing the charge radii is of the order of $0.01 \mathrm{fm}$, which is significantly better than that obtained with the HFB-17 and SLy4 EDFs. The excitation energies and $B(E 2)$ values of the first $2^{+}$states in the lead and tin isotopic chains also agree with the data much bet- 
ter than the results [24] obtained with the SLy4 and SKM* EDFs.

The SPEs of doubly magic nuclei are considered in more detail. The PC effects in SPEs are analyzed particularly. In addition to the usual pole diagram, the consideration includes the so-called tadpole term which is usually ignored [33, 34]. The latter is considered approximately, with neglecting the in-volume components of the vertex $g_{L}(r)$ of creating a surface $L$-phonon. This approximation works well in heavy nuclei, leading to a simple formula for the tadpole contribution which can be easily included into the calculation scheme. The tadpole contribution is often comparable with that of the pole diagram. As a rule, these two contributions to SPEs have different signs, their sum being often significantly less than that of the pole term alone. For the ${ }^{208} \mathrm{~Pb}$ nucleus, the calculation without $\mathrm{PC}$ corrections results in the average deviation $\left\langle\delta \varepsilon_{\lambda}\right\rangle_{\mathrm{rms}}$ from the experimental values equal to $0.51 \mathrm{MeV}$ for the Fayans EDF, whereas it is $1.15 \mathrm{MeV}$ for the HFB-17 EDF. With the PC corrections, including the tadpole contribution, we obtained very high accuracy: $\left\langle\delta \varepsilon_{\lambda}\right\rangle_{\mathrm{rms}}=0.34 \mathrm{MeV}$. For a comparison, the corresponding quantity, found in [33] within the RMF approach with PC corrections without the tadpole term, is $\left\langle\delta \varepsilon_{\lambda}\right\rangle_{\mathrm{rms}}=0.85 \mathrm{MeV}$.

To conclude, the Fayans EDF leads to a better agreement with the experimental data in all phenomena in spherical nuclei considered than several popular Skyrme EDFs chosen for a comparison. We relate it to the peculiarities of the Fayans EDF which reflect, in a hidden form, the energy dependence effects inherent to the selfconsistent TFFS.

\section{Acknowledgements}

The work was partially supported by the Grant NSh-932.2014.2 of the Russian Ministry for Science and Education and by the RFBR Grants 13-02-00085-a, 13-02-12106_ofi-m, 14-0200107-a, 14-22-03040_ofi-m.

\section{References}

[1] D. Vautherin and D.M. Brink, Phys. Rev. C 5, 626 (1972)

[2] W. Kohn and L.J. Sham, Phys. Rev. 140, A1133 (1965)

[3] P. Hohenberg and W. Kohn, Phys. Rev. 136 B864 (1964)

[4] J. Dechargé and D. Gogny, Phys. Rev. C 21, 1568 (1980)

[5] A.V. Afanasjev, S.E. Agbemava, D. Ray, and P. Ring, Phys. Rev. C 91, 014324 (2015)

[6] M. Baldo, P. Schuck, and X. Viñas, Phys. Lett. B 663, 390 (2008)

[7] M. Baldo, L.M. Robledo, P. Schuck, and X. Viñas, Phys. Rev. C 87, 064305 (2013)

[8] A.V. Smirnov, S.V. Tolokonnikov, and S.A. Fayans, Sov. J. Nucl. Phys. 48, 995 (1988)
[9] I.N. Borzov, S.A. Fayans, E. Kromer, and D. Zawischa, Z. Phys. A 355, 117 (1996)

[10] S.A. Fayans, JETP Lett. 68, 169 (1998)

[11] S.A. Fayans, S.V. Tolokonnikov, E.L. Trykov, and D. Zawischa, Nucl. Phys. A 676, 49 (2000)

[12] A.B. Migdal, Theory of finite Fermi systems and applications to atomic nuclei (Nauka, Moscow, 1965; transl. Wiley, New York, 1967)

[13] S.A. Fayans and V.A. Khodel, JETP Lett. 17, 444 (1973)

[14] E.E. Saperstein and V.A. Khodel, JETP 54, 12 (1981)

[15] V.A. Khodel, E.E. Saperstein, Phys. Rep. 92, 183 (1982)

[16] S.V. Tolokonnikov, I.N. Borzov, M. Kortelainen, Yu.S. Lutostansky, and E.E. Saperstein, J. Phys. G: Nucl. Part. Phys. 42, 075102 (2015)

[17] I.N. Borzov, E.E. Saperstein, and S.V. Tolokonnikov, Phys. At. Nucl. 71, 469 (2008)

[18] I.N. Borzov, E.E. Saperstein, S.V. Tolokonnikov, G. Neyens, and N. Severijns, Eur. Phys. J. A 45, 159 (2010)

[19] S.V. Tolokonnikov, S. Kamerdzhiev, D. Voytenkov, S. Krewald, and E.E. Saperstein, Phys. Rev. C 84, 064324 (2011)

[20] S.V. Tolokonnikov, S. Kamerdzhiev, S. Krewald, E.E. Saperstein, and D. Voitenkov, Eur. Phys. J. A 48, 70 (2012)

[21] S. Kamerdzhiev, S. Krewald, S. Tolokonnikov, E.E. Saperstein, and D.Voitenkov, EPJ Web of Conf. 38, 10002 (2012)

[22] E.E. Saperstein, S.V. Tolokonnikov, Phys. At. Nucl. 74, 1277 (2011)

[23] S.V. Tolokonnikov, S. Kamerdzhiev, S. Krewald, E.E. Saperstein, and D. Voitenkov, EPJ Web of Conf. 38, 04002 (2012)

[24] J. Terasaki, J. Engel, and G.F. Bertsch, Phys. Rev. C 78, 044311 (2008)

[25] H. Grawe, K. Langanke, and G. Martínez-Pinedo, Rep. Prog. Phys. 70, 1525 (2007)

[26] N.V. Gnezdilov, I.N. Borzov, E.E. Saperstein, and S.V. Tolokonnikov, Phys. Rev. C 89, 034304 (2014)

[27] S. Goriely, N. Chamel, and J. M. Pearson, Phys. Rev. Lett. 102, 152503 (2009)

[28] S. Goriely, http://www-astro.ulb.ac.be/bruslib/ nucdata/

[29] S.V. Tolokonnikov and E.E. Saperstein, Phys. At. Nucl. 73, 1684 (2010)

[30] D. Tarpanov, J. Dobaczewski, J. Toivanen, and B.G. Carlsson, Phys. Rev. Lett. 113, 252501 (2014)

[31] A. Bohr and B.R. Mottelson, Nuclear Structure (Benjamin, New York, 1974.), Vol. 2.

[32] A.P. Platonov, Sov. J. Nucl. Phys. 34, 612 (1981)

[33] E. Litvinova and P. Ring, Phys. Rev. C 73, 044328 (2006)

[34] Li-Gang Cao, G. Colò, H. Sagawa, and P.-F. Bortignon, Phys. Rev. C 89, 044314 (2014) 